\title{
Consideration and Exploration on the Demand Changes of Geographic Information in Smart City
}

\section{Xiaoli Liu ${ }^{\star}$ and Pengda Wu}

Chinese Academy of Surveying and Mapping, Beijing, China

\begin{abstract}
This article primarily describes the nature of geoinformatics, including the role of each of its components as understood today, and illustrates how the change in demand and the progress in science and technology are the two driving forces for the development of this subject. The changes in popular needs are next analyzed in detail, focusing on four aspects: the user group, intended usage, medium, and service. Lastly, it points out that geoinformatics work should be conducted following the development of geographic information.
\end{abstract}

\section{Publication History:}

Received: May 31, 2018

Accepted: September 27, 2018

Published: September 29, 2018

\section{Keywords:}

Flooding, SWAT, Sub-hourly, Bioretention, Permeable Pavement, Bankfull, Texas

\section{Introduction}

Service-oriented geoinformatics refers to the measurement, collection, and presentation of natural geographical features or surface man-made facilities in terms of shape, size, spatial location and attributes, as well as the processing and delivery of the acquired data as information and results. In essence, it is a comprehensive representation of the real world. The aforementioned tasks are primarily carried out by a group of people with professional expertise in real world objectives. The outputs are cartographic products that help in the scientific understanding of the world, without always having to go outdoors or practicing in person.

By initiating the concept of a 'smart city', new demands for geographic information are created. In particular, as cartography evolves from an analog medium to a digital one, geographic information accretes at unprecedented speed. The current increase in digital data accumulation has surpassed that from the past decades or even centuries of the analog era.

\section{Change in User Groups and Impact}

\section{Change in user group}

Professional land surveyors use special mapping tools to express physical measurements in a standardized map format and deliver these results to third-party users. These users are people with some knowledge of surveying and mapping, who can read and utilize the geographical information in these maps. These users usually work in GIS-dependent sectors such as land management, city planning, real estate management, water resource management, forestry, and transportation. Previously user groups were relatively limited but specific. The maps and geographic information required high precision and accurate depiction of specified properties, as departments would insert these various unique attributes onto their cartographic products [1]. They therefore did not demand any additional attributes to be included in their products. The surveying and mapping administration need only provide general map products that conform to the national and industrial standards, without concern for their applications, since GIS-dependent departments have the requisite knowledge, understanding, and skill to understand the principles and applications of cartographic products.
In recent years, with the acceleration of worldwide informatization and digitization, as highlighted by the statistical data of overseas authoritative survey agencies, there is almost no sector that can function without maps and geographic information. Application of such data therefore naturally expands from the strongly GISdependent departments to the weakly GIS-dependent ones, such as finance, insurance, industry and commerce, tax administration, the United Front Department, Development and Reform Commission, disciplinary inspection, Party committees, Women's Federations, and e-governments [2]. In today's world, users are no longer limited to professionals with a background knowledge of cartography, but are likely ordinary users lacking any professional background. Some users are those who lack the proficiency to read professional maps and geographic information, and are not very particular about high precision and accurate shape. They instead pay more attention to specific features of interest and attributes detailed by the maps and geographic information. The high precision data that surveying and mapping administrations have striven to provide, is naturally not the focus of the weakly GIS-dependent departments. The lack of map object attributes on the other hand, can create doubts on the credibility of the delivered products.

Some departments and enterprises attempt to bridge this gap by adding objects of potential interest and their attributes to the general maps and geographic information products to make government electronic maps, which usually meet the requirements of weakly GISdependent sectors, thereby achieving a twofold result. As China revises the security bottlenecks regarding maps and geographic information by technological means, the popularization of its use multiplies rapidly, as exemplified by use in the field of navigation. In a short span of ten years, electronic products with a navigation function, such as smart phones, cars, and home appliances, have become increasingly common. Global internet further renders billions of ordinary people

"Corresponding Author: Dr. Xiaoli Liu, Chinese Academy of Surveying and Mapping Beijing, China,100830; E-mail: liuxl@casm.ac.cn

Citation: Liu X, Wu P (2018) Consideration and Exploration on the Demand Changes of Geographic Information in Smart City. Int J Earth Environ Sci 3: 157. doi: https://doi.org/10.15344/2456-351X/2018/157

Copyright: (C) 2018 Liu et al. This is an open-access article distributed under the terms of the Creative Commons Attribution License, which permits unrestricted use, distribution, and reproduction in any medium, provided the original author and source are credited. 
Citation: Liu X, Wu P (2018) Consideration and Exploration on the Demand Changes of Geographic Information in Smart City. Int J Earth Environ Sci 3: 157. doi: https://doi.org/10.15344/2456-351X/2018/157

Page 2 of 5

loyal users of maps and geographic information. The traditional understanding and popular consensus for these products are challenged by the advancing accuracy, information content, presentation and storage mediums, as illustrated in Figure 1. The change in user groups offers vast business potentials, attracting internationally influential capital and enterprises to quickly enter the market, therefore constantly renewing map and geographic information products and services while exerting a subtle influence on the thinking and behavior within and outside the industry [3].

\section{Impact of user change}

As the users of maps and geographic information shift from professionals to the general public, not only has their number skyrocketed, but also caused the user group structure to undergo a qualitative change. This will undoubtedly lead to gradual changes in user demand that translate to substantial effects on cartography and geographic information development. In terms of content, the emphasis has moved from natural/physical characteristics to an inclusion of social attributes. In terms of precision, the demand also expands from that of one element to multiple elements. In short, as the user group increases from solely professionals to the general public, users become interested in numerous map objects and demand more attributes, while lowering the scrutiny on precision. The changes follow a pattern highlighted in Figure 2 .

\section{Opinions/ Impressions and interpretation}

Established by their name, surveying and mapping processes include the aggregation and representation of geographical data. When faced with user group change and the impact that ensues, simply surveying and mapping the location and shape of an object does not suffice. Locating map objects of user interest and defining their attributes has never been more important. As the old adage "survival of the fittest" goes, a number of modern enterprises keen to meet market demands, have broken tradition and risked a gambit amidst the doubt and

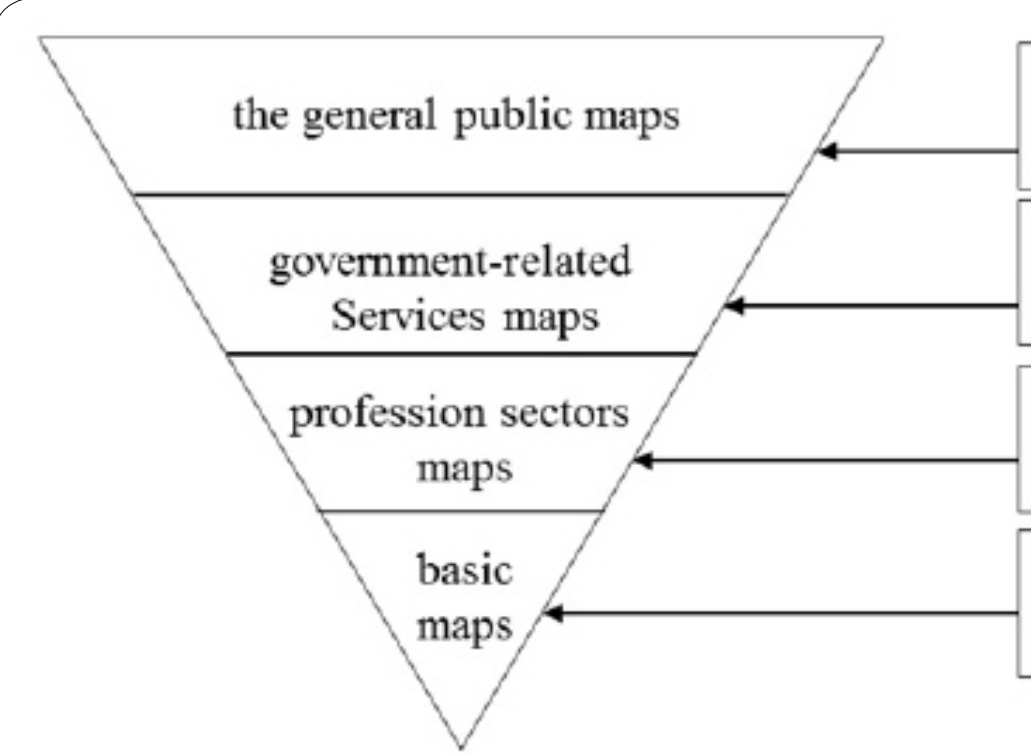

general public: Internet, navigation, traditional medium...

government: statistics bureau, public security, finance, tax administration...

profession sectors: city planning, land management, water resource management, transportation...

Figure 1: Changes in map and geographic information users.

topographic maps: 1:500, $1: 1000,1: 2000$..

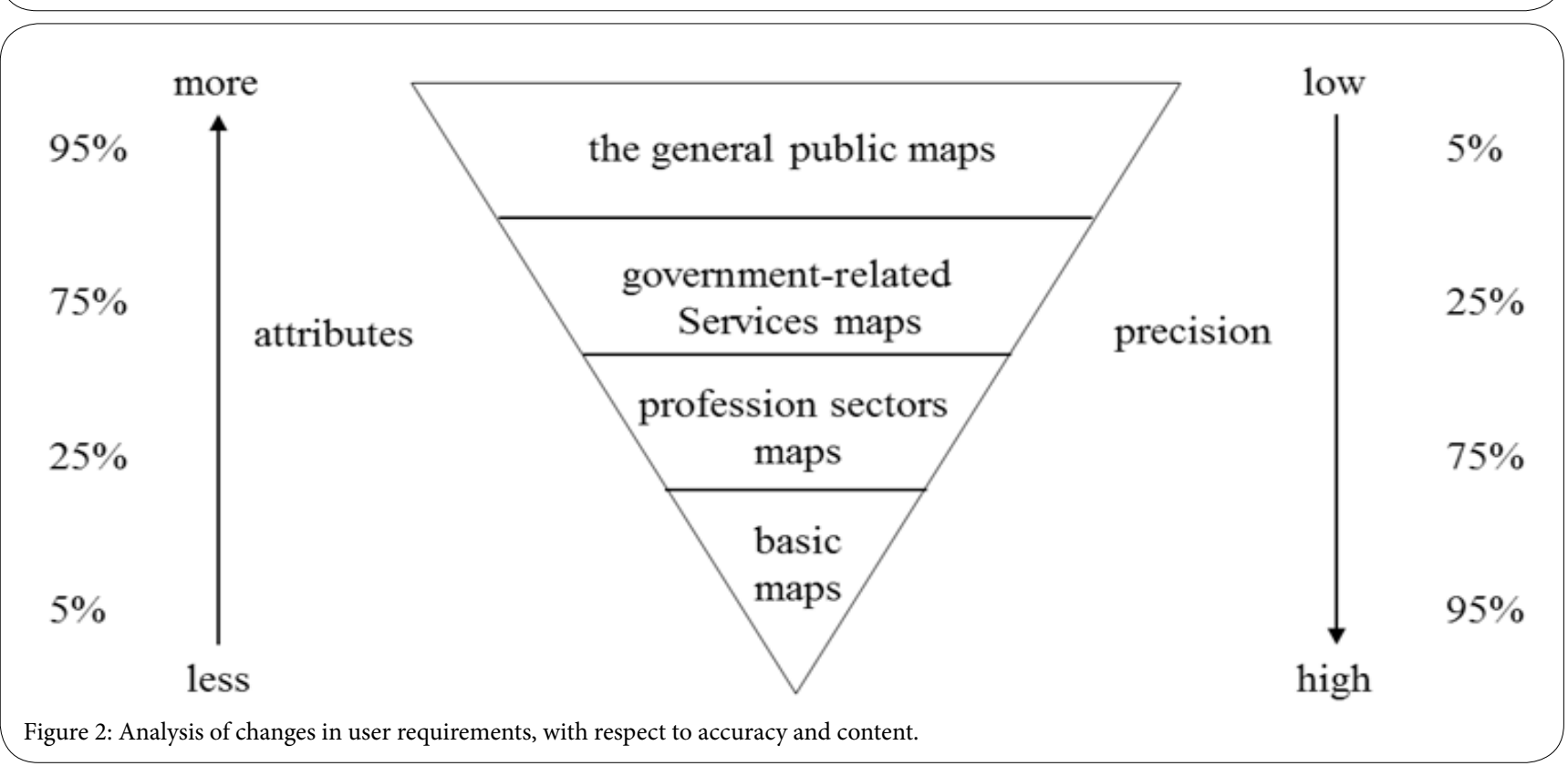


hesitation of traditional geoinformatics professionals. They research popular map objects and their attributes, and deliver generalized maps, based on common map and geographic information products, to meet the food, housing, transportation, tourism, shopping, and entertainment requirements of ordinary people. In just a few years, location-based map service products by companies such as AutoNavi, NavInfo, Careland and Tencent, have made geographic information readily available to the general public, and have earned the companies wide social applause for rapid development. Their employees have grown from dozens to thousands, and have become multinational companies in the United States, Hong Kong, Shenzhen, etc. The traditional surveying and mapping community on the other hand, is clamped in stereotype thinking and has almost no place in the emerging market of popular map services [4].

\section{Change in Intended Usage and itsIimpact}

\section{Change in intended usage}

Analog maps were initially used to reflect natural features such as topography, geomorphology, river systems, vegetation and soil, and carry specific content for land management, city planning, construction, water resource management, environmental protection, etc. They were blueprints used by professional departments compressed on an optimized space which could be easily carried around. With the inclusion of various highlights, they could be transformed into portable planning drafts and construction layout drawings. At this stage, maps served as references. On entering the digital age, analog maps are gradually replaced by geographical information systems, wherein thematic data could be arbitrarily stacked, ranging from a few layers to even hundreds of layers. Multiple attributes could be associated with representative geographical features. Compared to analog maps, the intended usage and function of geographic information is the spatial foundation for integrated thematic information. A major difference between the past and present is that a map product can only be owned and used by a single user each time. A geographic information product is simultaneously available to multiple users for a variety of spatial data, particularly with its integration to the internet of things (IoT). It has become a standard spatial reference for a multitude of thematic data, and is a benchmark for unifying this information in a spatial context, with an integrated platform for information stacking, and grounds for information sharing and exchange [5]. If information infrastructure is a highway, then geographic information is the one and only reliable and generally accessible vehicle on the road, carrying a variety of thematic information to all the network nodes. In short, analog maps are for the people, while digital geographic information is for the machines.

\section{Impact of change in use}

In the analog age, surveying and mapping personnel needed to both measure the physical world, and express it using lines and symbols. Before conducting any work on maps, technical personnel from the various fields would first need to master a map language that represents the physical world - map symbols and scales. They would need the ability to read a map, and mark it with the necessary information, creating a theme-specific chart based on the general map. During this period, the main motivation behind a map language was the convenience for a person to write, read and comprehend spatial information (e.g., elevation annotated by breaking a continuous topographic contour). The map symbols and rules were ultimately set for the visual comfort of people. In digital age, people began by digitizing analog maps. Although the medium changed, the essence of the maps did not. When the cartography community realized the limited value of the digitized analog maps, and the gradually increasing need for spatial analysis and efficient processing, data preparation for a geoinformation database, and its entire associated process, begin to take shape and dominance. The nature of this advancement was a revolution for traditional maps, reorienting them from human perspective to a machine one. The emergence of machine learning maps marks the transition from the 'digital age' to the 'age of intelligence.' As the development is driven largely by natural demand, and not a comprehensive top-level design from the stance of the intelligence age, data organization, presentation and analysis methods of the analog, digital and 'intelligent' age coexist today to suit users' needs. Therefore, the transition of a map from an analog to digital, and then to a computer compatible format, was an inevitable trend for the future development of geoinformatics technology.

\section{Opinions/ Impressions and interpretation}

Machines read maps quite differently from humans. In terms of scale, 1:500, 1:1000, 1:2000, etc., are denotations easily understandable for humans, who use basic arithmetic. However, continuing to use such representations in the binary (" 0,1 ") environment of computers, is not appropriate. Scales better suited for computer processing (in terms of 2n), such as 1, 4, 16, 64, 2561024 , etc., should be adopted. In terms of data organization, instantiated organization replaces the basic spatial data model of points, lines and surfaces. In terms of presentation, grids are replaced by a "pyramid" of map layers which facilitate machine understanding and recognition. Over the years, the surveying and mapping community, under the inertia of analog age thinking, never get rid of the deep-rooted influence of analog maps in the course of digitization. Instead, Google Maps, a multi-field newcomer, became a pioneer of the layered "pyramid" map on the internet, opening a new realm for online geoinformation service. With the demand for automation and intelligent technology rising, a systematic consideration of the theories and methods of the abstraction, expression, organization and analysis of geographic information on a new level, made the development of intelligent technology systems possible, accelerating the transition from digital to the 'intelligence' age.

\section{Change in Medium and Its Impact}

\section{Change in medium}

Geographic information has come a long way from being carved into tree barks, stones, and vessels of bronze and ceramic, to being plotted on paper. However, it remains the essentially same in nature, despite the change in medium. The concept involves a rich and vibrant three-dimensional representation on a plane or curved surface. Miniaturized two-dimensional drawings are used to represent the broad three-dimensional physical space, such as rows of mountains condensed into contour rings, or skyscrapers simplified into contoured bases and marked heights. Although means for expressing a three-dimensional world in two-dimensions has matured, it is still insufficient at times. After magnetic media became popular, maps and geographic information recording turned from analog to magnetic, which became the leading storage, transmission and exchange medium. Today, storage devices such as the compact discs, hard drives, flash drives, tape drives and blade servers are indispensable tools to people and businesses. 


\section{Impact of the change}

Being fully capable of representing and recording the physical word virtually, magnetic media broke the traditional practice of reducing dimensional space, such as the representation of a three-dimensional world in two dimensions. Undulating terrain, crisscrossing underground pipelines, and rows of buildings on the ground, can all be reproduced three-dimensionally in a digital environment. As the medium changed from analog to magnetic, it provided the tools and means for the expression of a three-dimensional world in three dimensions, and brought deep-rooted changes in mapping languages, making map symbols more visual, rules more complex, and effects more vivid.

\section{Opinions/Impressions and interpretation}

The scales and standard map expressions that were long known to people, have now lost their inherent meanings and functions in a 3D environment. In their place are new parameters, such as model levels (i.e., levels of detail) and viewing distance. Projection has also changed from a parallel projection to a central projection perspective. Height, distance, area and volume measurements are no longer in multiples of the map distance and actual distance as defined by a scale. However, the objects extracted from ground investigations, their expression, relation, etc., are still debated today when attempting a three-dimensional representation of the physical world. After actual construction, the market price for 3D models can vary by a factor of ten, reflecting the confusion, chaos, and the lack of a general standard in the industry. This is not surprising, as any change goes through a process of disorder before order. An early regulatory effort would be the common understanding, however a simple extension of two dimensional practices to three dimensions would not be appropriate. Instead, a standard view for the three-dimensional representation of the world should be adopted, borrowing traditions from twodimensional expressions, to re-examine and build systematic methods for cartographic terms and grammar, for a 3D manifestation.

\section{Change in Service Mode and its Impact}

\section{Change in service mode}

From the late 80 s to the early 90 s, with the support and dominance of information technology, such as computers and LAN, geoinformatics was successfully transformed from its analog phase to a digital one. Geoinformatics services have also evolved from providing maps to copy to the hard disk or floppy disk. Owing to time, batches, supplying units, user modification and many other factors, specialized applications based on geographic information emerged with a myriad of spatial foundations, becoming a "bottleneck" for informatization. In fields requiring an emergency response, comprehensive information integration, refined departmental management and macro-decision making, and inconsistencies in spatial foundations, make information integration time-consuming and laborious, with unreliable analysis results. Therefore, the establishment of a region-wide, continuous, unified, authoritative, and unique spatial foundation (or geospatial framework) is urgent and imperative requirement for the informatization of and development of local economy.

\section{Impact of the change}

There are several possible ways to construct a unified geographic information framework in a region. One is the centralized approach, relyingonfourmainstreamdatabases. Here, thegeographicinformation, as hard copies, is given to the appropriate department for data integration and compiled with other thematic data to serve external clients. However, as the geographic information, especially with elements closely related to economic and social development, such as transportation, places of residence, etc., changes rapidly, the needs of the government and its various departments (up-to-date data) cannot be met for the long-term, thus prompting updates and maintenance by geoinformatics professionals. Another approach is to continue by way of a traditional data delivery system, which is by using hard copies. As mentioned earlier, such a long-term practice will lead to inconsistencies in information databases. A team of global pioneers could use the wide area network and propose a distributed data storage platform, logical centralization, and a one-stop service for all geographic information. This concept is completely different from physical data centralization and the hard copy method mentioned above. To distinguish it from the past approaches and highlight its uniqueness, it can be coined the "public geographic information platform". Similar to a power grid, users turn switches on or off as needed, unperturbed by the electricity source, as it is supplied by a single strong power system and they need only pay as per usage. A public geographic information platform is essentially the same. Authorized users do not need to concern with the source of the geographic information, simply typing a URL would mobilize the various geographic information services, and re-develop the interface and functions.

\section{Opinions/ impressions and interpretation}

The replacement of traditional data with a public geographic information platform that provides online surveying and mapping services to users in the Internet age, is actually an effective separation of usership and ownership for geographic information. It first resolves the awkward situation of data dissemination within the surveying and mapping community from the analog age. Once data is distributed, its replication becomes difficult to verify, creating a dead end with a high payout and singular payback. Withholding data creates a race for acquisition from various sources by the user departments, peripheralizing the mapping and surveying community and its value. Secondly, this makes the information usage convenient, as the many users only have the usage rights, and need not pay for the ownership, thus largely reducing user costs while still having access to the latest data. Thirdly, this promotes information sharing, as different users can contribute their geospatial-related information to the same public geographic information platform, therefore eliminating information isolation due to disparities in the spatial foundations. This could multiply the value of information with respect to the price paid, as information is shared on a large scale.

\section{Conclusions}

Unprecedented changes in the demand for geoinformatics have occurred in recent years, exerting a profound influence on the conceptualization, expression, storage, management, service, and application of maps and geographic information. People working in this field need to look beyond the surface to trace the root and cause of the problem, make rational plans and grasp the key aspects, so as to understand thedevelopmentofcartographyandgeographicinformation in the information age and anticipate its future. The principles and methodologies of geospatial frameworks should be explored alongside concepts of a digital and 'smart city' to guide technological innovation and development of a spatio-temporal information platform, while inheriting and carrying forward past traditions. 
Citation: Liu X, Wu P (2018) Consideration and Exploration on the Demand Changes of Geographic Information in Smart City. Int J Earth Environ Sci 3: 157. doi: https://doi.org/10.15344/2456-351X/2018/157

Page 5 of 5

\section{Funding}

This research was supported by the National Surveying and Mapping Projects (A1705).

\section{Competing Interests}

The authors declare that no competing interests exist.

\section{References}

1. Surveying and Mapping Law of the People' s Republic of China (2007) Survey World.

2. Chengming Li, Xiaoli Liu, Jie Yin (2013) Investigation and Practice of Digital City to Smart City. J Bulletin of Surveying and Mapping.

3. Li Deren (2017) Investigation and Practice of Digital City to Smart City. J Acta Geodaetica et Cartographica Sinica 46: 1207-1212

4. Liu Xianlin (2017) Geographic Information in Mobile Internet Era. J Remote Sensing Information 32: 1-4

5. Li Deren, Yuan Y, Zhenfeng S (2014) Big Data in Smart City. J Geomatics and Information Science of Wuhan University 39: 631-640.

This article was originally published in a special issue:

Geographic Information Systems for Environmental Applications

Handled by Editor(s):

Dr. Antonio Martinez-Grana

Department of Geology

University of Salamanca

Spain

Dr. Federica Luca

Department of Earth Sciences

University of Calabria

Italy 